\title{
Um exemplar muito jovem de Mazama sp. encontrado na Gruta Cuvieri, região de Lagoa Santa, estado de Minas Gerais, Brasil
}

\author{
Artur Chahud ${ }^{1 *}$ \\ ${ }^{1}$ Laboratório de Estudos Evolutivos Humanos, Departamento de Genética e Biologia Evolutiva, Instituto de Biociências, Universidade de São Paulo, \\ São Paulo, SP, Brasil. \\ *E-mail para correspondência: arturchabud@yahoo.com
}

\begin{abstract}
RESUMO
Durante o Quaternário, as cavernas serviram como armadilhas naturais para numerosos grupos de animais e, devido às condições ambientais relativamente estáveis, a preservação nesse ambiente é melhor do que na superfície, de modo que o material osteológico encontrado nas cavernas representa um registro importante de eventos ambientais e mudanças na fauna. A Caverna Cuvieri faz parte do complexo Carste de Lagoa Santa, conhecido por seu grande número de cavernas contendo ossos preservados de animais recentes e extintos. Os cervídeos representam a família mais abundante de macrovertebrados encontrados na caverna de Cuvieri, com espécimes com grande variação ontogenética de representantes do gênero Mazama. O presente estudo descreve a ocorrência do espécime mais jovem deste gênero encontrado em um depósito de caverna. O espécime consiste em ossos apendiculares (fêmures, úmeros, tíbias, rádios, ulna e escápula) sem desgaste, ataque predatório ou evidência de intemperismo.
\end{abstract}

Palavras-chave: Carste de Lagoa Santa, Caverna, Cervidae, Mazama, Ontogenia, Quaternário

\begin{abstract}
A very young specimen from Mazama sp. found in Cuvieri Cave, Lagoa Santa region, State of Minas Gerais, Brazil. During the Quaternary, caves served as natural traps for numerous groups of animals and due to relatively stable environmental conditions preservation is better than on the surface, so the osteological material found in caves represents an important record of environmental events and faunal changes. Cuvieri Cave is part of the Lagoa Santa Karst complex, known for its large number of caves containing preserved bones of recent and extinct animals. Cervidae represent the most abundant family of macrovertebrates found at Cuvieri Cave, with specimens with large ontogenetic variation of representatives of the genus Mazama. The present study describes the occurrence of the youngest specimen of this genus found in a cave deposit. The specimen consists of appendicular bones (femurs, humerus, tibias, radios, ulna and scapula) without wear, predatory attack or evidence of weathering.
\end{abstract}

Keywords: Cave, Cervidae, Lagoa Santa Karst, Mazama, Ontogeny, Quaternary 


\section{INTRODUÇÃO}

A família Cervidae constitui um grupo de ungulados de grande diversidade taxonômica e ambiental que habita quase todos os continentes (sem registros na Antártida e Austrália). A origem na América do Sul é relativamente recente, pois ocorreu durante o Grande Intercâmbio Faunístico, 2,7 Ma (milhões de anos), e desde então foram registrados vários gêneros: Hippocamelus Leuckart, 1816; Blastocerus Wagner, 1844; Pudu Gray, 1852; Odocoileus Rafinesque, 1832 e Mazama Rafinesque, 1817, este último o mais comum (Duarte et al., 2012a).

O gênero Mazama possui 11 espécies reconhecidas, até o momento: M. rufina Bourcier \& Pucheran, 1852; M. bricenii Thomas, 1908; M. pandora Merriam, 1901; M. chunyi Hershkovitz 1959; M. americana Erxleben 1777; M. gouazoubira Fischer 1814; M. nana Hensel 1872; M. bororo Duarte 1996; M. tienhoveni Roosmalen 2015; M. nemorivaga Frédéric Cuvier, 1817 e M. temama Kerr, 1792 (Rossi, 2000; Vogliotti, 2003; Roosmalen, 2015).

O gênero é muito comum em boa parte do Neotrópico e se estende do sul do México à região norte central da Argentina. São pequenos macrovertebrados, com peso variando entre $15 \mathrm{~kg}$, em $M$. nana, a $65 \mathrm{~kg}$, em $M$. americana. Habitam diversos ambientes, desde florestas fechadas, alto de montanhas, cerrados ou savanas (Duarte et al., 2012a; 2012b; 2012c).

Durante o final do Pleistoceno (2,58Ma e 0011,7Ma) e todo o Holoceno (0011,7Ma até os dias atuais), diversas cavernas serviram como armadilhas para inúmeros grupos de animais e, devido às condições ambientais relativamente estáveis, a deterioração óssea é muito lenta se comparada com materiais osteológicos expostos em superfície, por isso os restos encontrado nas cavernas representam importante registro de eventos e mudanças faunísticas.

A Gruta Cuvieri faz parte do complexo do Carste de Lagoa Santa e foi alvo de inúmeros trabalhos de datação, espeleologia, paleoambientais, paleontológicos e sedimentológicos (Hubbe, 2008; Mayer, 2011; Hubbe et al., 2011; Haddad-Martim et al., 2017).

Em análise do material osteológico o gênero Mazama é o mais comum entre todos os restos de macrovertebrados encontrados na Gruta Cuvieri, sendo contabilizados mais de 60 indivíduos e é compreensível que existam animais de diferentes idades ontogenéticas. $O$ presente trabalho tem por objetivo ilustrar a presença de um indivíduo muito jovem, o mais novo encontrado na coleção da Gruta Cuvieri e fazer considerações sobre sua preservação e origem. 


\section{MATERIAIS E MÉTODOS}

A Gruta Cuvieri é uma pequena caverna desenvolvida na vertente nordeste de um pequeno maciço calcário, pertencente ao Carste de Lagoa Santa. Segundo Berbert-Born (2000) esta região possui um denso conjunto de feições geomorfológicas dissolutivas e uma hidrografia que pode ser caracterizada como mista de componentes fluviais (subaéreos) e cársticos (subterrâneos). As feições cársticas estão desenvolvidas em litótipos da Formação Sete Lagoas, Grupo Bambuí, Neoproterozóico, parte integrante do Cráton do São Francisco.

Segundo Hubbe et al. (2011) a Gruta Cuvieri localiza-se segundo as coordenadas UTM 23K 7846105/0603756 na região de Lagoa Santa, no estado de Minas Gerais, região sudeste do Brasil (Fig. 1). O sedimento encontrado na Gruta Cuvieri estava pouco consolidado, remobilizado ou modificado por fatores intempéricos externos, o que favoreceu a escavação e a exploração mais cuidadosa, com a obtenção de dados estratigráficos detalhados.

A Gruta Cuvieri é constituída de três pequenas cavidades verticais, armadilhas naturais, que foram denominadas Locus 1, 2 e 3 (Figura 1), com 16 metros, 4 metros e 8 metros de profundidade respectivamente, sendo que foram coletados material osteológico com rigoroso detalhamento estratigráfico dos Locus 2 e 3 (Hubbe et al., 2011).

O exemplar foi coletado no Locus 2 e é constituído de ossos apendiculares, fêmures, tíbias, úmeros, rádios e uma escápula (Figura 2).

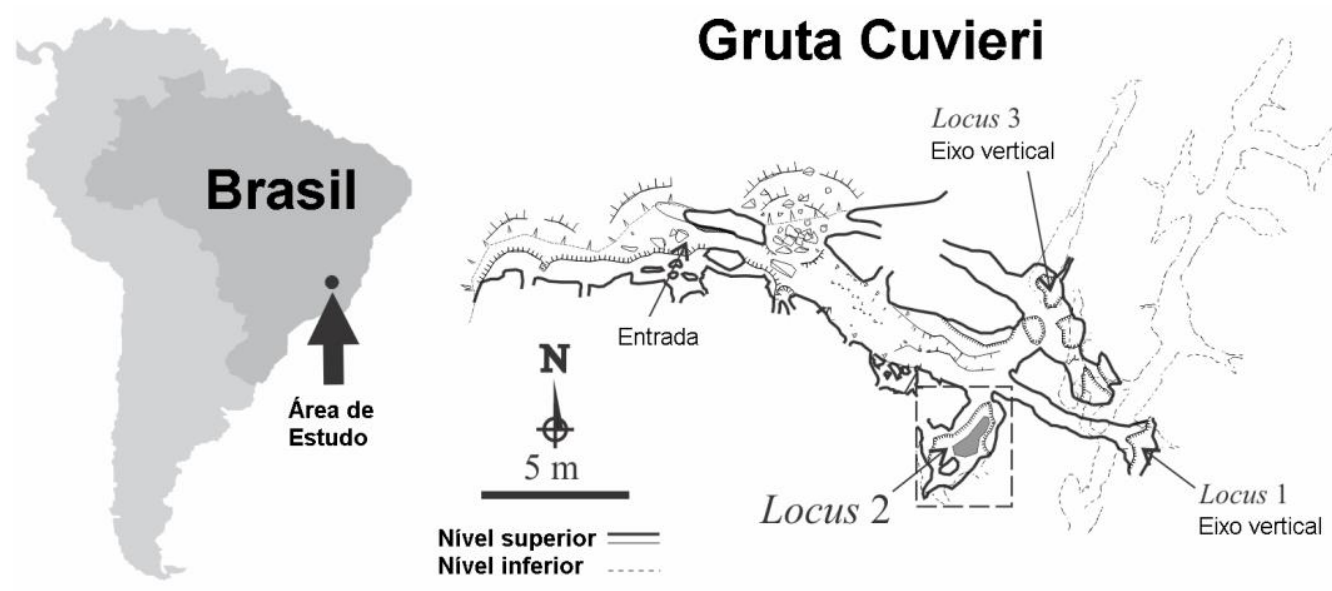

\section{Locus 2}

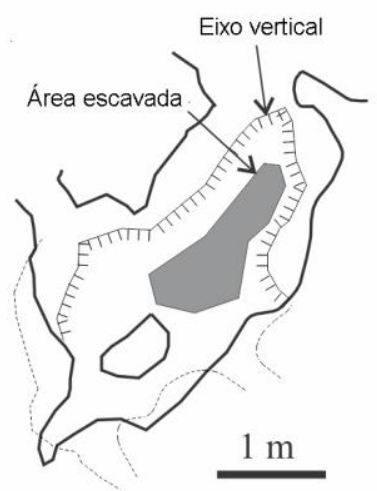

Figura 1. Localização geográfica da área de estudo, Região de Lagoa Santa e da Caverna Cuvieri, mostrando a posição do Locus 2 (mapa cortesia de Alex Hubbe e Grupo Bambuí de Pesquisas Espeleológicas para o Laboratório de Estudos Evolutivos Humanos (LEEH).

Segundo Hubbe et al. (2011) o Locus 2 é dividido em duas fácies. A primeira é constituída por um siltito marrom escuro, que constitui a fácies com maior quantidade de material osteológico (incluindo o 
espécime de estudo) e ocupa a maior parte do depósito explorado, e uma inferior de siltito marrom avermelhado, iniciando com $110 \mathrm{~cm}$ de profundidade de escavação e localizada na parte ocidental do Locus 2 (Hubbe et al., 2011, Fig. 6).

O espécime de estudo foi coletado entre os anos de 2002 e 2008, durante a pesquisa realizada pelo Professor Walter Neves, Origens e Microevolução do Homem na América, e estão depositados, numerados e registrados no Laboratório de Estudos Evolutivos Humanos (LEEH) do Instituto de Biociências da Universidade de São Paulo.

\section{RESULTADOS}

Classe Mammalia Linnaeus, 1758

Ordem Artiodactyla Owen, 1848

Família Cervidae Goldfuss, 1820

Subfamília Odocoileinae Pocock, 1923

Gênero Mazama Rafinesque, 1817

Mazama sp.

Figuras 2 e 3.

Localização geográfica e estratigráfica: Gruta Cuvieri, Carste de Lagoa Santa, Estado de Minas Gerais. Locus 2, entre 80-90cm de profundidade de escavação (Hubbe et al., 2011).

Material: O material é constituído de: fêmur esquerdo (CVL2-6182); fêmur direito (CVL2-6808), escápula esquerda (CVL2-6806), tíbia direita (CVL2-5366B), tíbia esquerda (CVL2-6183), úmero esquerdo (CVL2-6201), úmero direito (CVL2-5965), ulna direita (CVL2-6802), rádio direito (CVL2-6807) e rádio esquerdo (CVL2-6196). Todo o material coletado e estudado faz parte da coleção do Laboratório de Estudos Evolutivos Humanos (LEEH) do IB-USP.

Idade: O material estudado não pode ser datado, mas foi possível sugerir uma idade aproximada baseada no nível estratigráfico em que foi encontrado e nas datações obtidas para outros espécimes por Hubbe et al. (2011).

Entre os espécimes datados de níveis estratigráficos próximos incluem-se os pertencentes às famílias Tayassuidae, Cuniculidae e outros Cervidae, cujas datações obtidas em C14 (carbono 14) variaram entre 5 mil e 6 mil anos antes do presente, para a maioria dos espécimes. Apenas os animais extintos, Smilodon populator e Valgipes bucklandi alcançaram idades superiores a 10 mil anos e animais próximos da superfície obtiveram idades abaixo de 4 mil anos. O espécime estava localizado em níveis 
intermediários do locus 2 e, observando estas informações, foi possível especular que o espécime tenha entre 5 e 6 mil anos.

Tafonomia: Os ossos não apresentaram abrasão ou quebras significativas (observadas apenas na escápula e na ulna), se excluirmos o destacamento das epífises. O material (Figura 2) foi encontrado parcialmente articulado sendo que o fêmur esquerdo (CVL2-6182) estava em posição de articulação com a tíbia esquerda (CVL2-6183) e a escápula esquerda (CVL2-6806) radio direito (CVL2-6807) e ulna direita (CVL2-6802) estavam próximos, indicando que não houve significativa remobilização e o material foi enterrado ou protegido rapidamente, com mínimas perdas (Behrensmeyer, 1991).

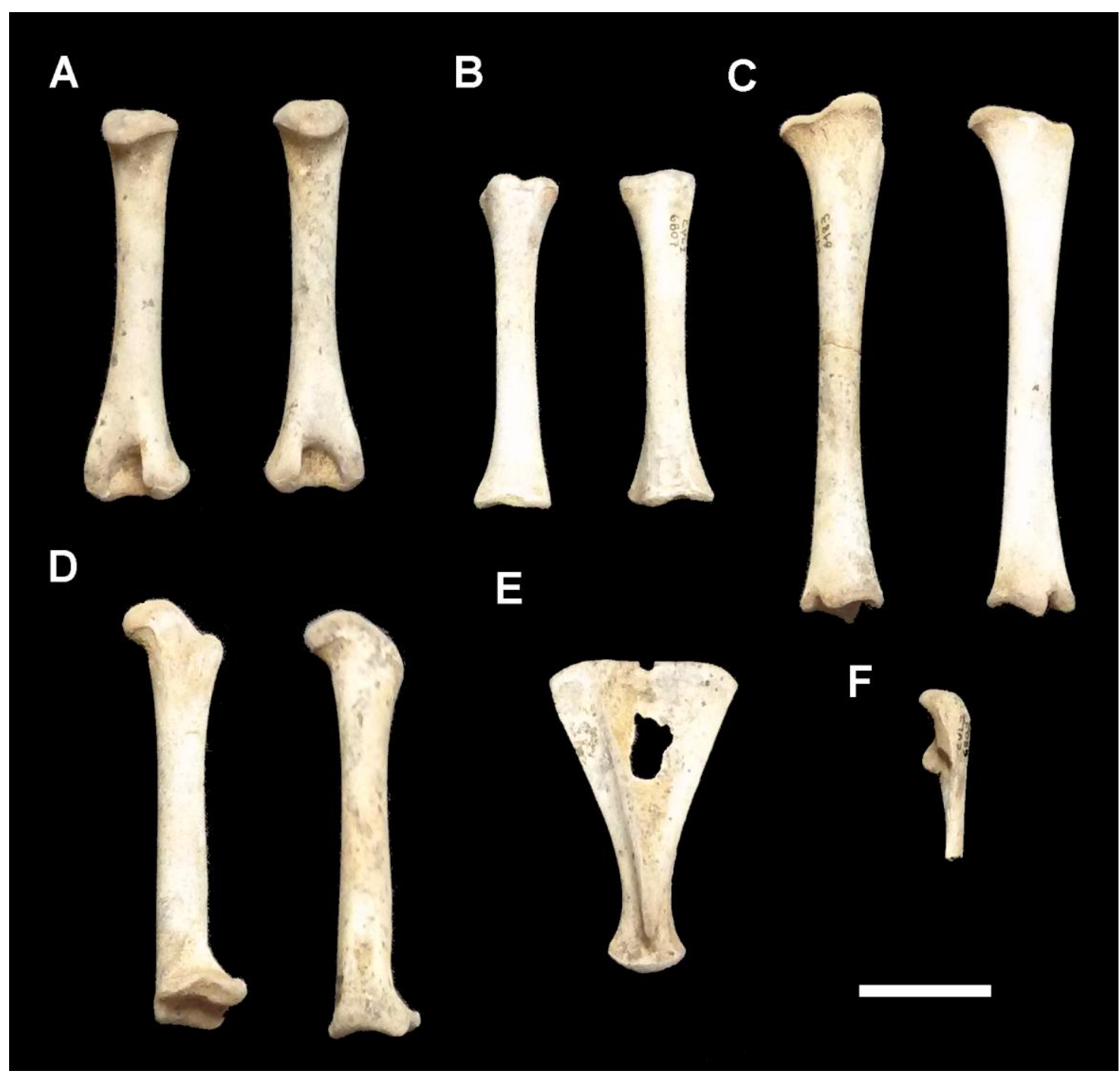

Figura 2. Ossos do indivíduo muito jovem de Mazama sp. A) úmero esquerdo (CVL2-6201) e úmero direito (CVL25965); B) rádio esquerdo (CVL2-6196) e rádio direito (CVL2-6807); C) tíbia esquerda (CVL2-6183) e tíbia direita (CVL2-5366B); D) fêmur esquerdo (CVL2-6182) e fêmur direito (CVL2-6808); E) escápula esquerda (CVL2-6806); F) ulna direita (CVL2-6802), Escala $20 \mathrm{~mm}$.

Como o ambiente era de uma caverna, a sedimentação pode ter sido muito mais lenta do que se estivesse em superfície, porém como as variações de temperatura e umidade são muito menores, o material teve maior proteção (Behrensmeyer, 1978; 1991). Não são registradas rachaduras de exposição intempéricas, reforçando a hipótese de pequeno período de exposição. 
Não foram caracterizados ataques de roedores ou carnívoros, reforçando a possibilidade de morte local e a proteção contra atividade biogênica de carniceiros. Ataques por roedores e carnívoros foram caracterizados em ossos maiores na Gruta Cuvieri no Locus 2 e 3 (Mayer, 2011). O pisoteamento também não ocorreu ou foi incipiente (afetando apenas os ossos menores e cranianos), uma vez que o material de maior porte está preservado (Figura 2).

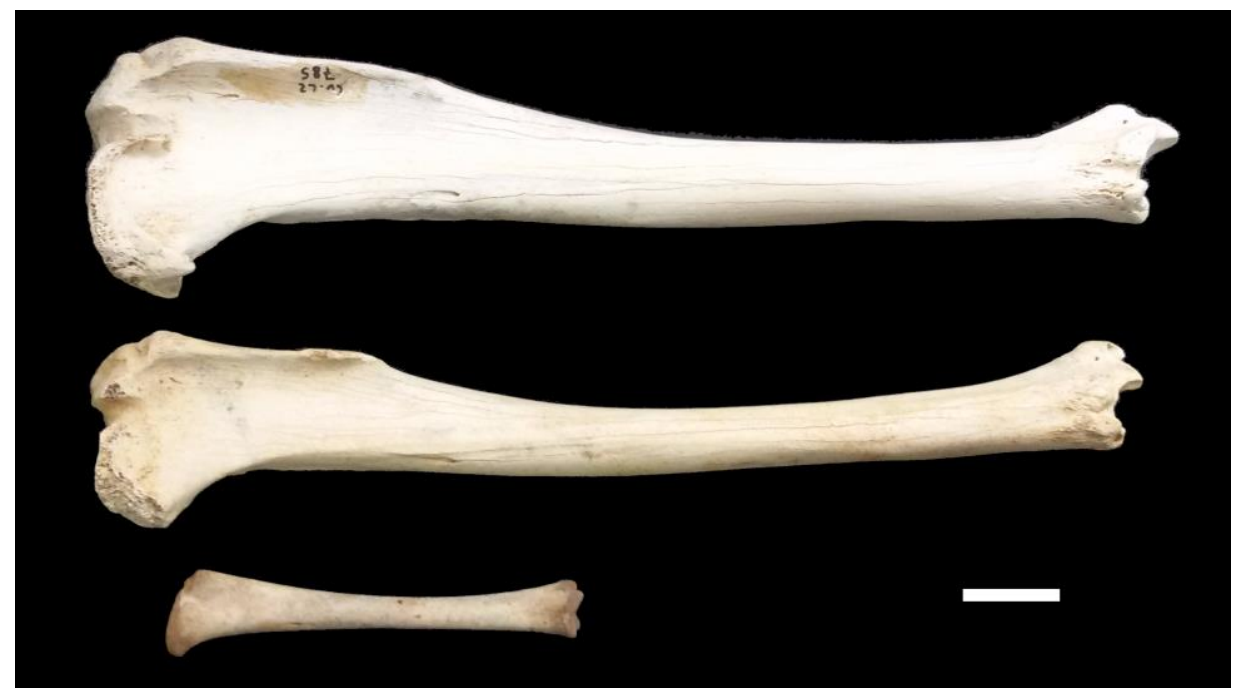

Figura 3. Comparação de tamanhos entre tíbias de espécies de Mazama encontrados na Gruta Cuvieri. Acima Mazama cf. americana, no meio Mazama cf. gouazoubira e abaixo o indivíduo muito jovem. Escala $20 \mathrm{~mm}$.

\section{DISCUSSÃO}

Como esperado para um animal muito jovem e de pequenas dimensões, o espécime não tem nenhuma epífise fusionada ou pequenos ossos completos preservados. Apenas os ossos maiores puderam ser identificáveis, enquanto ossos menores ou mais frágeis foram destruídos com o passar dos anos.

As pequenas quebras na escápula e na ulna representam partes muito finas ou frágeis e que seriam facilmente perdidas com o tempo.

Em nenhum osso foram observadas marcas musculares visíveis ou significativas, um indicativo de que era muito jovem. O local onde as epífises deveriam estar posicionadas não possui sinais de esforço e estão arredondadas e lisas.

Estudos com cervos da Gruta Cuvieri registrou duas espécies de Cervidae, Mazama cf. americana e Mazama cf. gouazoubira. A análise do tamanho dos ossos (Tabela 1) ao ser comparado com ossos de indivíduos adultos das duas espécies de Mazama encontrados na Gruta Cuvieri (Figura 3), leva a assumir-se que o espécime é muito pequeno, com proporções próximas a de um recém-nascido ou muito jovem (Loponte et al., 2019). 
Tabela 1. Medidas obtidas dos ossos do espécime encontrado na Gruta Cuvieri. Técnica utilizada para medições usada por Von de Driesch (1976) e Loponte et al. (2019).

\begin{tabular}{|c|c|c|c|}
\hline Número & Osso & Parte medida & Medidas (mm) \\
\hline \multirow[t]{5}{*}{ CVL2-6182 } & Fêmur & Maior comprimento & 69,74 \\
\hline & esquerdo & Profundidade da extremidade proximal & 9,40 \\
\hline & & Profundidade da extremidade distal & 14,60 \\
\hline & & Maior largura da extremidade proximal & 15,60 \\
\hline & & Maior largura da extremidade distal & 14,40 \\
\hline \multirow[t]{5}{*}{ CVL2-6808 } & Fêmur & Maior comprimento & 69,90 \\
\hline & direito & Profundidade da extremidade proximal & 10,00 \\
\hline & & Profundidade da extremidade distal & 14,30 \\
\hline & & Maior largura da extremidade proximal & 16,50 \\
\hline & & Maior largura da extremidade distal & 14,30 \\
\hline \multirow[t]{3}{*}{ CVL2-6806 } & Escápula & Maior comprimento & 47,40 \\
\hline & esquerda & Maior comprimento do processo articular (glenóide) & 11,80 \\
\hline & & Maior largura do processo articular (glenóide) & 9,00 \\
\hline \multirow[t]{5}{*}{ CVL2-5366b } & Tíbia & Maior comprimento & 82,10 \\
\hline & direita & Profundidade da extremidade proximal & 14,90 \\
\hline & & Profundidade da extremidade distal & 10,10 \\
\hline & & Maior largura da extremidade proximal & 16,90 \\
\hline & & Maior largura da extremidade distal & 13,90 \\
\hline \multirow[t]{5}{*}{ CVL2-6183 } & Tíbia & Maior comprimento & 83,30 \\
\hline & esquerda & Profundidade da extremidade proximal & 12,90 \\
\hline & & Profundidade da extremidade distal & 9,90 \\
\hline & & Maior largura da extremidade proximal & 19,10 \\
\hline & & Maior largura da extremidade distal & 13,90 \\
\hline \multirow[t]{5}{*}{ CVL2-6201 } & Úmero & Maior comprimento & 58,20 \\
\hline & esquerdo & Profundidade da extremidade proximal & 14,50 \\
\hline & & Profundidade da extremidade distal & 9,10 \\
\hline & & Maior largura da extremidade proximal & 11,10 \\
\hline & & Maior largura da extremidade distal & 15,50 \\
\hline \multirow[t]{5}{*}{ CVL2-5965 } & Úmero & Maior comprimento & 59,00 \\
\hline & direito & Profundidade da extremidade proximal & 14,90 \\
\hline & & Profundidade da extremidade distal & 8,90 \\
\hline & & Maior largura da extremidade proximal & 11,90 \\
\hline & & Maior largura da extremidade distal & 15,20 \\
\hline CVL2-6802 & Ulna direita & Maior largura da extremidade proximal & 10,30 \\
\hline \multirow[t]{5}{*}{ CVL2-6807 } & Rádio & Maior comprimento & 53,80 \\
\hline & direito & Profundidade da extremidade proximal & 8,10 \\
\hline & & Profundidade da extremidade distal & 9,70 \\
\hline & & Maior largura da extremidade proximal & 11,80 \\
\hline & & Maior largura da extremidade distal & 12,90 \\
\hline \multirow[t]{5}{*}{ CVL2-6196 } & Rádio & Maior comprimento & 53,30 \\
\hline & esquerdo & Profundidade da extremidade proximal & 7,90 \\
\hline & & Profundidade da extremidade distal & 9,80 \\
\hline & & Maior largura da extremidade proximal & 11,90 \\
\hline & & Maior largura da extremidade distal & 13,10 \\
\hline
\end{tabular}




\section{CONSIDERAÇÕES FINAIS}

Os Cervidae da Gruta Cuvieri foram pouco estudados taxonomicamente, sendo considerados em trabalhos anteriores apenas como Mazama sp. ou Cervidae indeterminados (Hubbe, 2008; Mayer, 2011; Hubbe et al., 2011). No entanto, a partir do estudo com os ossos encontrados, foi possível constar pelo menos duas espécies (Figura 3) e variações ontogenéticas.

Observando que as duas espécies identificadas possam ser comparáveis aos atuais Mazama americana e M. gouazoubira segundo Duarte et al. (2012a e 2012b), ambas possuem gestação de sete meses e, no caso de $M$. gouazoubira, os filhotes ficam escondidos na mata nas primeiras semanas e se tornam independentes da mãe com aproximadamente 8 meses de vida.

O espécime é muito pequeno e tem proporções similares a de filhotes recém-nascidos ou ao de um feto próximo do nascimento de ambas as espécies de Mazama. No entanto a hipótese do feto pode ser descartada por não haver um adulto associado aos seus ossos e sim apenas ossos pequenos.

Não há evidências de que a ocorrência do exemplar tenha sido oriunda de atividade predatória, devido à ausência de marcas de mordidas ou quebras, e que foi resultado de queda acidental no Locus 2 , como ocorrido na maioria dos animais de médio e grande porte da Gruta Cuvieri (Hubbe, 2008).

\section{AGRADECIMENTOS}

O autor agradece a Professora Doutora Mercedes Okumura responsável pelo Laboratório de Estudos Evolutivos Humanos do Instituto de Biociências da Universidade de São Paulo, onde a coleção osteológica Guajá está depositada e aos pesquisadores que coletaram o material da Gruta Cuvieri e que forneceram informações importantes para a realização deste trabalho.

\section{REFERÊNCIAS BIBLIOGRÁFICAS}

Berbert-Born, M. 2000. Carste de Lagoa Santa In: C. Schobbenhaus, D.A.; Campos, E.T.; Queiroz, M.; Winge, M.; Berbert-Born (Eds.). Sítios Geológicos e Paleontológicos do Brasil. 16p.

Behrensmeyer, A.K. 1978. Taphonomic and ecologic information from bone weathering. Paleobiology 4(2): $150-162$.

Behrensmeyer, A.K. 1991. Terrestrial Vertebrate Accumulations. Taphonomy: In: Allison, P.A. \& D.E.G. 
Briggs, (eds.). Taphonomy: releasing the data locked in the fossil record. New York, Plenum Press. p.291- 335.

Duarte, J.M.B.; Vogliotti, A.; Zanetti, E.S.; Oliveira, M.L.; Tiepolo, L.M.; Rodrigues, L.F. \& Almeida, L.B. 2012a. Avaliação do Risco de Extinção do Veado-catingueiro Mazama gouazoubira G. Fischer [Von Waldhein], 1814, no Brasil. G. Fischer [von Waldhein], 1814, no Brasil. Biodiversidade Brasileira 1: $50-$ 58.

Duarte, J.M.B.; Vogliotti, A.; Zanetti, E.S.; Oliveira, M.L.; Tiepolo, L.M.; Rodrigues, L.F. \& Almeida, L.B. 2012b. Avaliação do Risco de Extinção do veado-mateiro Mazama americana Erxleben, 1777, no Brasil. Biodiversidade Brasileira 1: 33-41.

Duarte, J.M.B.; Abril, V.V.; Vogliotti, A.; Zanetti, E.S.; Oliveira, M.L.; Tiepolo, L.M.; Rodrigues, L.F. \& Almeida, L.B. 2012c. Avaliação do Risco de Extinção do veado cambuta Mazama nana Hensel, 1872, no Brasil. Biodiversidade Brasileira 1: 59-67.

Haddad-Martim, P.M.; Hubbe, A.; Giannini, P.C.F.; Auler, A.S.; Piló, L.B.; Hubbe, M.; Mayer, E.; Wang, X.; Cheng, H.; Edwards, R.L. \& Neves, W.A. 2017. Quaternary depositional facies in cave entrances and their relation to landscape evolution: The example of Cuvieri Cave, eastern Brazil. Catena 157: 372-387.

Hubbe, A. 2008, Contextualização taxonômica, tafonômica e morfométrica dos remanescentes ósseos da megamastofauna da gruta Cuvieri (MG) um sitio paleontológico do pleistoceno tardio. São Paulo. Dissertação (Mestrado em Genética e Biologia Evolutiva), Instituto de Biociências da USP. 141p.

Hubbe, A.; Haddad-Martim, P.M.; Hubbe, M.; Mayer, E.L.; Strauss, A.; Auler, A.S.; Piló, L.B. \& Neves, W.A., 2011a. Identification and importance of critical depositional gaps in pitfall cave environments: the fossiliferous deposit of Cuvieri Cave, eastern Brazil. Palaeogeography, Palaeoclimatology, Palaeoecology 312: $66-78$.

Loponte, D.; Corriale, M.J.; Mucciolo, L. \& Acosta, A. 2019. Sex and age-biased exploitation and metric characterization of medium-sized deer in the lower Paraná wetland, South America. International Journal of Osteoarchaeology: 1-27.

Mayer, E.L. 2011. Processos de formação de um depósito fossilifero, um abismo na Gruta Cuvieri (MG): Taxonomia, Tafonomia e distribuição espacial. São Paulo. Dissertação (Mestrado em Ecologia). Instituto de Biociências, USP. 145p.

Roosmalen, M.G.M. 2015. Hotspot of new megafauna found in the Central Amazon: the lower Rio Aripuanã Basin. Biodiversity Journal 6 (1): 219-244. 
Rossi, R. V. 2000. Taxonomia de Mazama Rafinesque, 1817 do Brasil (Artiodactyla, Cervidae). Dissertação (Mestrado em Ciências Biológicas). Universidade de São Paulo. 174p.

Vogliotti, A. 2003. História natural de Mazama bororo (Artiodactyla; Cervidae) através da etnozoologia, monitoramento fotográfico e rádio-telemetria. Dissertação (Mestrado em Ecologia Aplicada). Universidade de São Paulo. Escola Superior de Agricultura “Luiz de Queiroz". 99p.

Von de Driesch, A. 1976. A guide to the measurement of animals bones from archaeological sites. Peabody Museum Bolletin. 138p. 